\title{
Predictors of Greater Vaping Dependence and Higher Vaping Frequencies among Canadian Youth and Young Adults over 12-Month
}

\author{
Tianru Wang ${ }^{1}$, Safa Ahmad ${ }^{1}$, Robert Schwartz ${ }^{1,2}$, Michael Chaiton ${ }^{1,2}$ and Susan Bondy ${ }^{1,2}$ \\ ${ }^{1}$ Dalla Lana School of Public Health, University of Toronto, Toronto, Canada \\ ${ }^{2}$ Ontario Tobacco Research Unit, University of Toronto, Toronto, Canada \\ E-mail: tianru.wang@mail.utoronto.ca
}

Received October 28, 2020

Accepted for publication December 23, 2020

Published online September 5, 2021

\begin{abstract}
Background: The prevalence of electronic cigarette use has doubled among Canadian youth and young adults in recent years. However, little is known about the predictors of greater vaping nicotine dependence. This study identifies variables associated with greater Penn-State Electronic Cigarette Dependence Index (PS-ECDI) scores and higher vaping sessions per month (SPM) among this population.
\end{abstract}

Methods: Data were drawn from a longitudinal study that recruited 1048 Canadian participants aged 16-26 in 2018. Quota sampling was used to ensure enough regular e-cigarette users were recruited. The current study restricted analyses to the 459 participants who were baseline vapers and have completed both the baseline and 12-month follow-up surveys. Linear regression analyses were employed, with the use of the best subset modelling strategy to obtain reduced models.

Results: Baseline vapers who were $\geq 18$ and married or cohabiting, had used other tobacco products, had 30 or more puffs per vaping session, started vaping at an earlier age, vaped to quit/reduce smoking, and had used a disposable cigarette-like vaping device and/or an advanced box or tubular device and/or a pod vape in the last 6-month were associated with greater PS-ECDI scores compared to their respective counterparts. Additionally, baseline vapers who were $\geq 18$ and married or cohabiting, had used cannabis, had 30 or more puffs per vaping session; vaped to reduce/quit smoking and/or because friends vape; had used a pod vape in the last 6-month were more likely to have higher SPM at the 12-month follow-up compared to their respective counterparts.

Conclusion: $11 \%$ of the participants were classified as high vaping nicotine dependence and $42 \%$ of the participants had increased their SPM over 1 year. The findings provide targets for vaping reduce or cessation programs and potential policy change in the regulation and sales of pod vape. 